\title{
A computational and literature-based evaluation for a combination of chiral anti-CoV drugs to block and eliminate SARS-CoV-2 safely
}

Mohd. Suhail ${ }^{a *}$

\author{
aDepartment of Chemistry, Jamia Millia Islamia, (A Central University) Jamia Nagar, New \\ Delhi-110025, India.
}

\begin{abstract}
It has been a great challenge for scientists to develop an anti-covid drug/vaccine with fewer side effects, since the coronavirus began. Of course, the prescription of chiral drugs (chloroquine or hydroxychloroquine) has been proved wrong because these chiral drugs neither kill the virus nor eliminate it from the body, but block SARS-CoV-2 from binding to human cells. Another hurdle in front of the world, is not only the positive test of the patient recovered from coronavirus but also the second wave of Covid 19. Hence, the word demands such a drug or drug combination which not only prevents the entry of SARS-CoV-2 in the human cell but also eliminates it or its material from the body completely. The presented computational study explains (i) why the prescription of chiral drugs was not satisfactory (ii) what types of modification can make their prescription satisfactory (iii) the mechanism of action of chiral drugs (chloroquine and hydroxychloroquine) to block SARS-CoV-2 from binding to human cells, and (iv) the strength of mefloquine to eliminate SARS-CoV-2. As the main protease ( $\left.\mathrm{M}^{\text {pro }}\right)$ of microbes is considered as an effective target for drug design and development, the binding affinities of mefloquine with the main protease $\left(\mathrm{M}^{\mathrm{pro}}\right)$ of JC virus and SARS-CoV-2, were calculated, and then compared to know the eliminating strength of mefloquine against SARS$\mathrm{CoV}-2$. The main protease $\left(\mathrm{M}^{\text {pro }}\right)$ of JC virus was taken because mefloquine has already shown a tremendous result of eliminating it from the body. The current study includes the docking results and literature data in support of the prescription of a combination of S-(+)-hydroxychloroquine and $(+)$ mefloquine. Besides, the presented study also confirms that the prescription of only hydroxychloroquine would not be so effective as in combined form with mefloquine.
\end{abstract}

Keywords: Chiral drugs, S-(+)-hydroxychloroquine, (+) mefloquine, computational study and SARS-CoV-2.

*Correspondence to [suhailchem.786@gmail.com] 


\section{Introduction}

To find a suitable drug or vaccine for the coronavirus, has become a great challenge for the researchers in this modern and advanced era. Virologists, microbiologists, clinicians, industrialists, and government authorities are working very hard day-night. Besides, numerous antiviral drugs were employed for the treatment of SARS-CoV-2 infection [1], but an acceptable solution did not come in front of the world yet. Chloroquine blocking the coronavirus from binding to human cells [2], was considered as a suitable drug, but conditions became very critical, when chloroquine was prescribed to the patient fighting with coronavirus (https://edition.cnn.com/2020/03/23/africa/chloroquine-trump-nigeria-intl/index.html). After that, hydroxychloroquine was suggested for the same, because it shows fewer side effects[3], but the question of causing side effects, remained unsolved, which has made the prescription of these two drugs failed. Hence, one question, why chloroquine and hydroxychloroquine did not give satisfactory results in vivo, arises here. The main reason behind it, is the prescription of these chiral drugs in the racemic form i.e. enantiomeric form. The main problem is that each enantiomeric form has its own biological activity with different mechanisms [4-14]. Hence, in the case of chiral drugs, the property of existing in different forms, makes the conditions very much complicated, and their mechanism of action also. Therefore, this is a great challenge for the researchers to find out the most biologically active enantiomeric form $[15,16]$. Nobody knows, which enantiomeric form of chloroquine and hydroxychloroquine caused side effects. Because of the prescription in the racemic form, the mechanism of action of chloroquine and hydroxychloroquine to block the coronavirus remains poorly understood, which the presented study explains for the first time.

Besides the mechanism of action and the side effects of chloroquine and hydroxychloroquine, the most important and notable thing standing in front of the world, is the positive report of the patient recovered from the coronavirus [https://curlytales.com/somerecovered-coronavirus-patients-are-testing-positive-for-it-again/]. All around the world, there have been several cases of people recovering from COVID-19 only to later test positive again. This report clearly shows the existence of coronavirus or its material in the body [https://www.healthline.com/health-news/people-reinfected-with-covid-19-werent-infectious].

Moreover, the existence of coronavirus or its material in the recovered patient may also the cause of the second wave of this pandemic which has come in India 
[https://timesofindia.indiatimes.com/india/first-vs-second-wave-of-covid-19-in-india-things-you-needto-know/articleshow/82143427.cms] and other countries [https://time.com/5954416/india-covidsecond-wave/]. This thing raises many questions whose unsolved chemistry has created another obstacle in front of the world. The presented study also resolves this problem. Having read the research papers regarding the virus elimination from the body, it was found that mefloquine has been used for the same. Mefloquine has given the tremendous results in vitro [17] as well as in vivo [18]. The virus eliminated through mefloquine from the body, was JC virus. Hence, mefloquine has played an important role in the elimination of JC virus from the body completely [18], but the problems of causing side effects, with this drug, were the same. It was just because of the prescription of this chiral drug in the racemic form. Mefloquine is presently manufactured and vended as a racemate of the (R,S)- and $(S, R)$-enantiomers by Hoffman-LaRoche, a Swiss pharmaceutical company. It is on the WHO's List of Important Drugs, the harmless and most active drugs desirable in a health system [19]. Hence, if mefloquine is prescribed for the removal of SARS-CoV-2 from the body, we have to face many questions such as experimental data, theoretical or computational data supporting the idea of prescription of this drug. Besides, this drug is chiral in nature, and can not be prescribed as it is i.e. in the racemic form. Therefore, another question related to the prescription of a biologically active enantiomeric form of mefloquine to avoid the toxicities, side effects, and other problems, also arises here. The presented study also resolves this question.

Of course, the drugs and their targets should be known, when the mechanism of action, and the strength of the drug, is being studied. Hence, a docking study was done using the enantiomers of the chiral drugs taken in the current study, as the ligands, while other things (such as main protease) required to inhibit the microbes [20], were selected as the targets. The experimental support in choosing SARS-CoV-2 $\mathrm{M}^{\text {pro }}$ as the drug target, is the involvement of this protein in the regulation of replication and transcription [21,22]. Moreover, during drug development strategy, the residues of the targets (such as SARS-CoV-2 $\mathrm{M}^{\mathrm{pro}}$ )[20] involving in the interactions with the drugs, assist as a platform for the development of potent and selective inhibitors of microbes. Hence, keeping these facts into consideration, the main proteases $\left(\mathrm{M}^{\text {pros }}\right)$ of both JC virus and SARS-CoV-2, were interacted with mefloquine computationally and then compared. Also, the enantiomers of only hydroxychloroquine were also interacted computationally with the main protease of SARS-CoV-2 just to compare the binding affinities 
with those of mefloquine so that it can be recognized if only a single enantiomeric form of hydroxychloroquine could work or not.

\section{Materials and Methods}

\subsection{Software's and Tools}

All the software and tools used for studying the interaction between enantiomers and targets, are Discovery Studio Visualizer, MarvinSketch (16.9.12 version), LigPlot, AutoDock Vina 4.2, Protein Data Bank (PDB), MGL tools, PubChem and PyMOL.

A computational study is very helpful not only in understanding the reaction mechanism [2327] but also in the drug development [28] and mechanism of action in pharmacokinetics [29]. There were three steps to be followed for the current computational evaluation. The first step was the preparation of pdb files of targets and ligands, the second was the molecular docking simulation, and the final step was the data analysis. The experiments done on chloroquine [2] and hydroxychloroquine [3], were evaluated computationally, to know the mechanism of action of both chloroquine and hydroxychloroquine in blocking SARS-CoV-2, while the literature data was used to know the most biologically active enantiomer of both chloroquine and hydroxychloroquine. In the same way, the experiments done on mefloquine[18,17] for the elimination of JC virus, were also evaluated computationally, and compared with the computationally evaluated docking results for SARS-CoV-2, to know the eliminating strength of mefloquine against it.

\subsection{Receptor/Target Preparation}

First, all the pdb files of targets with pdb code: 6lzg for SARS-CoV-2 with its receptor; $6 \mathrm{m03}$ for SARS-CoV-2 M $\mathrm{M}^{\text {pro; }} 5 \mathrm{j} 40$ for JC virus $\mathrm{M}^{\text {pro }}$ (Figure 1a), (Figure 1b) and (Figure 1c) respectively, were obtained from the protein data bank (www.rcsb.org). All the pdb files obtained were not pure due to the presence of impurities such as ligands and water molecules. Hence, all the pdb files of the targets were made pure by removing the impurities attached to the targets, using Discovery Studio Visualizer so that it could be used for further study. After that, the pdb files were opened one by one in AutoDock Tools (ADT) 4.2 [30] to add the non-polar hydrogen atoms, followed by Gasteiger charges assigned to targets. After the addition of all the things mandatory for the simulation study, the pdb files of targets were saved as pdbqt format. 


\subsection{Ligands (Enantiomers) Preparation}

In the ligands (enantiomers) preparation, MarvinSketch was used. All the enantiomers of chloroquine, hydroxychloroquine, and mefloquine taken in the present study, were saved in pdb files (Figure 1d), (Figure 1e) and (Figure 1f), respectively. All the pdb files of ligands (enantiomers of chloroquine, hydroxychloroquine, and mefloquine), like receptor preparation, were converted into pdbqt format one by one using AutoDock Tools (ADT) 4.2 [30], and the docking was achieved by using ADT considering all the rotatable bonds of the ligand as rotatable, and the receptor as rigid[30]. The grid box size of $60 \times 80 \times 110 \mathrm{~A}^{\circ}$ with $0.375 \mathrm{~A}^{\circ}$ spacing, was used.

\subsection{Docking Methods}

All the files of enantiomers, formatted as pdbqt, were docked with targets one by one using AutoDock vina[31] program. For the docking method, the coordinates of the source were set at $\mathrm{x}=30.054, \mathrm{y}=22.75$, and $\mathrm{z}=4$ 4.171. Many autonomous docking runs were applied for each ligand (enantiomer) and targets to find the lowest free energy of binding confirmation from the largest cluster.

\subsection{Data and Analysis}

The analysis of the number of hydrogen bonds, the residues of enantiomers as well as targets involved in hydrogen bondings, mode of interaction, and bond lengths of hydrogen bonds, were studied by PyMOL. On the other hand, LigPlot 1.4.5 [32] was used for the study of enantiomeric hydrophobic interactions with the targets.

\section{Results}

The docking studies of the enantiomers of chloroquine, hydroxychloroquine and mefloquine with the targets, were performed. The results are given in Table 1 . The representative enantiomeric interactions of chloroquine and hydroxychloroquine with SARS-CoV-2 spike attached to its receptor, are shown in Figure 2a and Figure 2b, respectively, while those of mefloquine with JC virus main protease $\left(\mathrm{M}^{\text {pro }}\right)$ as well as SARS-CoV-2 main protease $\left(\mathrm{M}^{\text {pro }}\right)$, are shown in Figure 3 and Figure 4a, respectively. Besides, the representative enantiomeric interactions of hydroxychloroquine with SARS-CoV-2 $\mathrm{M}^{\text {pro }}$ are shown in Figure 4b. These figures show clearly that the enantiomers interacted with the targets differently. The most 
significant and notable point in the docking results (Table 1), was the attachment of enantiomers only with the receptor of SARS-CoV-2, not with its spike.

The binding affinities of each enantiomer of chloroquine and hydroxychloroquine with the receptor of SARS-CoV-2, were the same $(-4.2 \mathrm{kcal} / \mathrm{mol})$. In the case of chloroquine, R-form of chloroquine did not form any hydrogen bond, while S-form of chloroquine formed 1-H bond with the receptor of SARS-CoV-2 spikes. In the same way, the number of hydrogen bonds was different in both enantiomers of hydroxychloroquine. It was observed that R-form of hydroxychloroquine formed 3-H bonds, while S-form of hydroxychloroquine did not form any hydrogen bond with the receptor of SARS-CoV-2 spikes. On the other hand, the binding affinities of the enantiomers of mefloquine with SARS-CoV-2 $\mathrm{M}^{\text {pro }}$ and JC virus $\mathrm{M}^{\text {pro }}$, were very shocking, because the binding affinities of mefloquine with SARS-CoV-2 $\mathrm{M}^{\text {pro }}$, were greater than those with JC virus $\mathrm{M}^{\text {pro }}$. The binding affinities ranged from -3.5 to $-3.6 \mathrm{kcal} / \mathrm{mol}$ in the case of JC virus $\mathrm{M}^{\text {pro }}$, while these ranged from -6.8 to $-7.3 \mathrm{kcal} / \mathrm{mol}$ in the case of SARS-CoV-2 $\mathrm{M}^{\text {pro }}$. On the other hand, the binding affinities of hydroxychloroquine with SARS-CoV-2 $\mathrm{M}^{\text {pro }}$ were lesser as compared to those of mefloquine (table 1). In the case of JC virus $\mathrm{M}^{\text {pro }}$, RR-form of mefloquine formed 2-H bonds; RS-form formed no hydrogen bond; SR-form formed 2-H bonds; SS-form formed only 1-H bond. Besides, with SARS-CoV-2 M $\mathrm{M}^{\text {pro }}$, RR-form of mefloquine formed 2-H bonds; RS-form formed no hydrogen bond; SR-form formed only 1-H bond; SSform formed only 1-H bond.

Additionally, enantiomeric hydrophobic interactions of chloroquine and hydroxychloroquine with the receptor of SARS-CoV-2 spike, were also seen as shown in Figure 5a and Figure 5b, respectively, while those of mefloquine with JC virus $\mathrm{M}^{\text {pro }}$ and SARS-CoV-2 $\mathrm{M}^{\text {pro }}$, are shown in Figure 6 and Figure 7a, respectively. The enantiomeric hydrophobic interactions of hydroxychloroquine with SARS-CoV-2 $\mathrm{M}^{\text {pro }}$ are shown in Figure $7 \mathrm{~b}$. The common residues involved in enantiomeric hydrophobic interaction of chloroquine and hydroxychloroquine with the receptor of SARS-CoV-2 spikes, were Ala533(A), Asp543(A), His535(A), Lys416(A), Ser545(A), Glu430(A), Glu536(A), Asn546(A), His535(A), Lys416(A), Lys534(A), Ser547(A), while those in mefloquine with JC virus $\mathrm{M}^{\text {pro }}$, were Gln339(A), Gln340(A), Ser336(A), Trp271(A), OD2, Asn333(A), Asp343(A), OG; with SARS-CoV-2 Mpro, were Leu287(A), Leu286(A), Met276(A), Tyr239(A), Tyr237(A), Thr199(A), Asp289(A), Thr199(A). The common residues involved in enantiomeric hydrophobic interaction of 
hydroxychloroquine with SARS-CoV-2 $\mathrm{M}^{\text {pro }}$, were Leu 271(A), Leu 272(A), Leu286(A), Leu287(A), Met276(A), Tyr237(A), Thr199(A), Arg131(A), Asp289(A), Glu288(A), Glu290(A).

\section{Discussion}

Of course, the results were very complicated but intriguing as well as exciting. The interpretation was done with the help of docking results, literature data, and experiments done on the presented drugs by others previously. Definitely, the interpretations will play an important role in the solution of the coronavirus pandemic. The enantiomers of chloroquine and hydroxychloroquine interacted with the receptor of SARS-CoV-2 spikes, in different ways/fashions, that is why the number of hydrogen bonds, was found different in different enantiomers. The similar binding affinities of chloroquine and hydroxychloroquine with the receptor of SARS-CoV-2 spikes, show that these drugs bind to the receptor of SARS-CoV-2 spikes with equal strength. The attachment of the enantiomers of chloroquine and hydroxychloroquine only with the receptor of SARS-CoV-2 spikes, not with SARS-CoV-2 spike, was too much helpful in understanding the mechanism of action of both the drugs. Actually, it means that both the drugs bind with the receptor of SARS-CoV-2 spikes, that is why SARS-CoV-2 does not recognize its receptor, and become unable to enter in the human cell (Figure 8). In this way, the mechanism of blocking coronavirus from binding to human cells, is resolved.

Obviously, the notable results were observed in the interaction/binding study of mefloquine with both JC virus $\mathrm{M}^{\text {pro }}$ and SARS-CoV-2 $\mathrm{M}^{\text {pro }}$. The different enantiomers of mefloquine interacted with both the targets in different modes, that is why the number of hydrogen bonds, was found different in different enantiomers of mefloquine. The most important and notable thing was the binding affinities of each enantiomer of mefloquine, which were greater for SARS$\mathrm{CoV}-2 \mathrm{M}^{\text {pro }}$ than those for JC virus $\mathrm{M}^{\text {pro }}$. Since the binding affinities of the enantiomers of hydroxychloroquine with SARS-CoV-2 $\mathrm{M}^{\text {pro }}$ were not greater than those of mefloquine, the prescription of only hydroxychloroquine would not be so effective as in combined form with mefloquine. Hence, this thing helped too much in understanding the strength of mefloquine for the elimination of SARS-CoV-2 from the body. The diverse binding affinities of the enantiomers of the reported chiral drugs with the targets, were observed due to their dissimilar stereochemical 
configuration. The docking studies showed that the connections among the enantiomers and targets, were due to hydrophobic interactions as well as hydrogen bonding. The strength of the interaction was based on both binding affinities and the number of hydrogen bonds. Hence, in the coronavirus pandemic, the presented study may prove mefloquine to be a milestone for the elimination of coronavirus from the body, as per docking results.

Now, another question arising on the most biologically active enantiomeric form of the respective chiral drugs, is also resolved with the help of literature data. As we know that the chiral drugs such as chloroquine and hydroxychloroquine, both have one chiral center, and exist in two enantiomeric forms that is why the prescription of these drugs in the racemic form, raises many questions related to their side effects. It is already confirmed that hydroxychloroquine is better than chloroquine[33][3]. Now, one question, which enantiomeric form of hydroxychloroquine having less toxicity and side effect, should be prescribed to the patient, arises here. The most biologically active enantiomer of hydroxychloroquine having has less toxicity and side effect, is S-(+)-hydroxychloroquine [34] which has already been patented as an active and effective form [35]. It means that ' $\mathrm{R}$ ' enantiomer of hydroxychloroquine is inactive and causes side effects, that must be avoided. Hence, except the racemic form of hydroxychloroquine, S-(+)-hydroxychloroquine would be a suitable and effective drug in blocking the coronavirus, with less toxicity and side effect. On the other hand, as per docking results, all the enantiomers of mefloquine interacted with SARS-CoV-2 M pro more strongly, as compared with JC virus $\mathrm{M}^{\text {pro }}$, showing the higher capability to eliminate SARS-CoV-2 from the body. In the prescription of mefloquine, conditions become more complicated. The main reason behind it, is the presence of two chiral centers in mefloquine, which confirms the existence of mefloquine in four enantiomeric forms. Hence, the prescription of mefloquine in the racemic form for the elimination of SARS-CoV-2, will raise same questions, as in the case of hydroxychloroquine. The most biologically active enantiomer of mefloquine, that could be prescribed, is (+)-mefloquine, which has already been confirmed as an active and effective form[36]. WRAIR has published several papers outlining ongoing efforts to make mefloquine safer by producing only (+)-mefloquine because this enantiomer has not only less affinity towards plasma but also a shorter half-life than other enantiomeric forms [36]. Hence, (+)mefloquine would be a suitable and effective drug in eliminating SARS-CoV-2 from the body with less toxicity as well as side effect as per both docking results and literature data [36]. Based 
on the docking results and literature data $[2,3,17,18,36]$, the prescription of a combination of S$(+)$-hydroxychloroquine and (+)-mefloquine, must be suitable for the safest treatment of coronavirus. In these two drugs, S-(+)-hydroxychloroquine would be able to block the coronavirus from binding to human cells, while $(+)$-mefloquine would be able to eliminate it from the body, with less side effect.

\section{Conclusion}

After a long discussion based on the modeling results, literature data in support of the less toxicity of S-(+)-hydroxychloroquine \& (+)-mefloquine, and the work done by others previously, I found that chloroquine and hydroxychloroquine, both have the capability to block the SARSCoV-2 from binding to human cells, but not to eliminate it from the body. Of course, in these two drugs, only hydroxychloroquine would be suitable to be prescribed, but the prescription of its only S-(+)-form, would make it more suitable and effective with fewer side effects. On the other hand, as per docking results, mefloquine may exhibit its capability to eliminate the SARSCoV-2 from the body, as mefloquine did in the case of JC virus. The prescription of its only (+)form, would make it more suitable and effective with less side effect. Therefore, the current computational study may prove the prescription of a combination of S-(+)-hydroxychloroquine and (+)-mefloquine to be a troubleshooter in this pandemic situation. Moreover, the prescription of only a single enantiomeric form will also be very helpful in understanding the mechanism of action of both chiral drugs. Hence, the presented study may be acceptable to the scientific community.

\section{Acknowledgment}

I am very thankful to my guide, Professor Imran Ali, Chemistry Department, Jamia Millia Islamia (A Central University), New Delhi, Delhi, India for guiding and supporting morally.

\section{Funding Source}

There is no funding source for this manuscript. 


\section{References}

[1] A. Zumla, J.F.W. Chan, E.I. Azhar, D.S.C. Hui, K.-Y. Yuen, Coronaviruses — drug discovery and therapeutic options, Nat. Rev. Drug Discov. 15 (2016) 327-347. doi:10.1038/nrd.2015.37.

[2] M. Wang, R. Cao, L. Zhang, X. Yang, J. Liu, M. Xu, Z. Shi, Z. Hu, W. Zhong, G. Xiao, Remdesivir and chloroquine effectively inhibit the recently emerged novel coronavirus (2019-nCoV) in vitro, Cell Res. 30 (2020) 269-271. doi:10.1038/s41422-020-0282-0.

[3] J. Liu, R. Cao, M. Xu, X. Wang, H. Zhang, H. Hu, Y. Li, Z. Hu, W. Zhong, M. Wang, Hydroxychloroquine, a less toxic derivative of chloroquine, is effective in inhibiting SARS-CoV-2 infection in vitro, Cell Discov. 6 (2020) 16. doi:10.1038/s41421-020-01560 .

[4] I. Ali, M.N. Lone, M. Suhail, Z.A. Al-Othman, A. Alwarthan, Enantiomeric resolution and simulation studies of four enantiomers of 5-bromo-3-ethyl-3-(4-nitrophenyl)piperidine-2,6-dione on a Chiralpak IA column, RSC Adv. 6 (2016) 14372-14380. doi:10.1039/c5ra26462f.

[5] I. Ali, M. Suhail, Z.A. AL-Othman, A. Alwarthan, H.Y. Aboul-Enein, Enantiomeric resolution of multiple chiral centres racemates by capillary electrophoresis, Biomed. Chromatogr. 30 (2016) 683-694. doi:10.1002/bmc.3691.

[6] I. Ali, M. Suhail, M.N. Lone, Z.A. Alothman, A. Alwarthan, Chiral resolution of multichiral center racemates by different modalities of chromatography, J. Liq. Chromatogr. Relat. Technol. 39 (2016) 435-444. doi:10.1080/10826076.2016.1152582.

[7] I. Ali, M. Suhail, Z.A. Alothman, A. Alwarthan, Chiral separation and modeling of baclofen, bupropion, and etodolac profens on amylose reversed phase chiral column, Chirality. 29 (2017) 386-397. doi:10.1002/chir.22717.

[8] I. Ali, M. Suhail, L. Asnin, Chiral separation of quinolones by liquid chromatography and capillary electrophoresis, J. Sep. Sci. 40 (2017) 2863-2882. doi:10.1002/jssc.201700200.

[9] I. Ali, M. Suhail, L. Asnin, H.Y. Aboul-Enein, Reverse elution order of $\beta$-blockers in chiral separation, J. Liq. Chromatogr. Relat. Technol. 40 (2017) 435-441. 
doi:10.1080/10826076.2017.1327443.

[10] I. Ali, M. Suhail, H.Y. Aboul-Enein, Chiral analysis of macromolecules, J. Liq. Chromatogr. Relat. Technol. 41 (2018) 749-760. doi:10.1080/10826076.2018.1514509.

[11] I. Ali, M. Suhail, H.Y. Aboul-Enein, Advances in chiral multidimensional liquid chromatography, TrAC Trends Anal. Chem. 120 (2019) 115634. doi:10.1016/j.trac.2019.115634.

[12] I. Ali, M. Suhail, Z.A. ALOthman, A.M. Al-Mohaimeed, A. Alwarthan, Chiral resolution of four stereomers and simulation studies of newly synthesized antibacterial agents having two chiral centers, Sep. Purif. Technol. 236 (2020) 116256. doi:10.1016/j.seppur.2019.116256.

[13] M. Suhail, I. Ali, Gas chromatography : A tool for drug analysis in biological samples, Chem. Int. 6 (2020) 277-294. doi:10.5281/zenodo.3735676.

[14] Z.A. ALOthman, A.G. ALanazi, M. Suhail, I. Ali, HPLC enantio-separation and chiral recognition mechanism of quinolones on Vancomycin CSP, J. Chromatogr. B. (2020) 122335. doi:10.1016/j.jchromb.2020.122335.

[15] M.F. Alajmi, A. Hussain, M. Suhail, S.D. Mukhtar, D.R. Sahoo, L. Asnin, I. Ali, Chiral HPLC Separation and Modeling of Four Stereomers of DL-Leucine-DL-Tryptophan Dipeptide on Amylose Chiral Column, Chirality. 28 (2016) 642-648. doi:10.1002/chir.22624.

[16] I. Ali, M. Suhail, Z.A. Alothman, A.Y. Badjah, Stereoselective interactions of profen stereomers with human plasma proteins using nano solid phase micro membrane tip extraction and chiral liquid chromatography, Sep. Purif. Technol. 197 (2018) 336-344. doi:10.1016/j.seppur.2018.01.029.

[17] M. Brickelmaier, A. Lugovskoy, R. Kartikeyan, M.M. Reviriego-Mendoza, N. Allaire, K. Simon, R.J. Frisque, L. Gorelik, Identification and Characterization of Mefloquine Efficacy against JC Virus In Vitro, Antimicrob. Agents Chemother. 53 (2009) 1840-1849. doi:10.1128/AAC.01614-08.

[18] T.E. Gofton, A. Al-Khotani, B. O’Farrell, L.C. Ang, R.S. McLachlan, Mefloquine in the treatment of progressive multifocal leukoencephalopathy, J. Neurol. Neurosurg. Psychiatry. 82 (2011) 452-455. doi:10.1136/jnnp.2009.190652.

[19] World Health Organization (2019). World Health Organization model list of essential 
medicines: 21st list 2019. Geneva: World Health Organization. hdl:10665/325771. WHO/ MVP/EMP/IAU/2019.06. License: CC BY-NC-SA 3.0 IGO

[20] L. Zhang, D. Lin, X. Sun, U. Curth, C. Drosten, L. Sauerhering, S. Becker, K. Rox, R. Hilgenfeld, Crystal structure of SARS-CoV-2 main protease provides a basis for design of improved $\alpha$-ketoamide inhibitors, Science (80-. ). (2020) eabb3405. doi:10.1126/science.abb3405.

[21] H. Yang, M. Yang, Y. Ding, Y. Liu, Z. Lou, Z. Zhou, L. Sun, L. Mo, S. Ye, H. Pang, G.F. Gao, K. Anand, M. Bartlam, R. Hilgenfeld, Z. Rao, The crystal structures of severe acute respiratory syndrome virus main protease and its complex with an inhibitor, Proc. Natl. Acad. Sci. 100 (2003) 13190-13195. doi:10.1073/pnas.1835675100.

[22] K. Anand, Structure of coronavirus main proteinase reveals combination of a chymotrypsin fold with an extra alpha-helical domain, EMBO J. 21 (2002) 3213-3224. doi:10.1093/emboj/cdf327.

[23] S.M. Somagond, M.N. Wari, S.K.J. Shaikh, S.R. Inamdar, M.K. Shankar, D.J. Prasad, R.R. Kamble, Detailed analytical studies of 1,2,4-triazole derivatized quinoline, Eur. J. Chem. 10 (2019) 281-294. doi:10.5155/eurjchem.10.4.281-294.1844.

[24] M.A. Shanshal, Q.A. Yusuf, C-C and C-H bond cleavage reactions in acenaphthylene aromatic molecule, an ab-initio density functional theory study, Eur. J. Chem. 10 (2019) 403-408. doi:10.5155/eurjchem.10.4.403-408.1889.

[25] V. Darugar, M. Vakili, S.F. Tayyari, F.S. Kamounah, R. Afzali, Application of Hammett equation to intramolecular hydrogen bond strength in para-substituted phenyl ring of trifluorobenzoylacetone and 1-aryl-1,3-diketone malonates, Eur. J. Chem. 9 (2018) 213221. doi:10.5155/eurjchem.9.3.213-221.1713.

[26] B.K. Al-Salami, Microwave synthesis of some N-phenylhydrazine-1-carbothioamide Schiff bases, Eur. J. Chem. 9 (2018) 74-78. doi:10.5155/eurjchem.9.2.74-78.1673.

[27] M. Suhail, S.D. Mukhtar, I. Ali, A. Ansari, S. Arora, Theoretical DFT study of Cannizzaro reaction mechanism: A mini perspective, Eur. J. Chem. 11 (2020) 139-144. doi:10.5155/eurjchem.11.2.139-144.1975.

[28] A. Malik, S. Afaq, B. El-Gamal, M. Abd Ellatif, W.N. Hassan, A. Dera, R. Noor, M. Tarique, Molecular docking and pharmacokinetic evaluation of natural compounds as targeted inhibitors against Crz1 protein in Rhizoctonia solani, Bioinformation. 15 (2019) 
277-286. doi:10.6026/97320630015277.

[29] P. Stenberg, C.A.S. Bergstr??m, K. Luthman, P. Artursson, Theoretical Predictions of Drug Absorption in Drug Discovery and Development, Clin. Pharmacokinet. 41 (2002) 877-899. doi:10.2165/00003088-200241110-00005.

[30] G.M. Morris, R. Huey, W. Lindstrom, M.F. Sanner, R.K. Belew, D.S. Goodsell, A.J. Olson, AutoDock4 and AutoDockTools4: Automated docking with selective receptor flexibility, J. Comput. Chem. 30 (2009) 2785-2791. doi:10.1002/jcc.21256.

[31] O. Trott, A.J. Olson, AutoDock Vina: Improving the speed and accuracy of docking with a new scoring function, efficient optimization, and multithreading, J. Comput. Chem. (2009) NA-NA. doi:10.1002/jcc.21334.

[32] R.A. Laskowski, M.B. Swindells, LigPlot+: Multiple Ligand-Protein Interaction Diagrams for Drug Discovery, J. Chem. Inf. Model. 51 (2011) 2778-2786. doi:10.1021/ci200227u.

[33] D.S. Finbloom, K. Silver, D.A. Newsome, R. Gunkel, Comparison of hydroxychloroquine and chloroquine use and the development of retinal toxicity., J. Rheumatol. 12 (1985) 692-4. http://www.ncbi.nlm.nih.gov/pubmed/4057189.

[34] I. D'Acquarica, I. Agranat, Chiral switches of chloroquine and hydroxychloroquine: potential drugs to treat COVID-19, Drug Discov. Today. 25 (2020) 1121-1123. doi:10.1016/j.drudis.2020.04.021.

[35] William Francis, Michne Vera Johanna. Use of (S)-(+)-Hydroxychloroquine. European Patent Office. EP0588430B1 (1994)

[36] P. Schlagenhauf, Mefloquine for Malaria Chemoprophylaxis 1992-1998: A Review, J. Travel Med. 6 (1999) 122-133. doi:10.1111/j.1708-8305.1999.tb00843.x. 